Check for updates

Cite this: J. Mater. Chem. A, 2021, 9, 19886

Received 18th March 2021

Accepted 20th May 2021

DOI: 10.1039/d1ta02262h

rsc.li/materials-a

\section{Understanding multi-scale battery degradation with a macro-to-nano zoom through its hierarchy $\dagger$}

\author{
Guibin Zan, $t^{\mathrm{a}}$ Jin Zhang, $t^{\mathrm{a}}$ Federico Monaco, $t^{\mathrm{b}}$ Sheraz Gul, ${ }^{\mathrm{c}}$ Guannan Qian, ${ }^{\mathrm{a}}$ \\ Jizhou Li, ${ }^{a}$ David J. Vine, ${ }^{\mathrm{c}}$ Peter Cloetens, (D) ${ }^{\mathrm{b}}$ Wenbing Yun, ${ }^{\mathrm{C}}$ Piero Pianetta ${ }^{\mathrm{a}}$ \\ and Yijin Liu (DD *a
}

\begin{abstract}
Lithium-ion batteries (LIBs) feature structural and chemical complexities across a broad range of length scales. It is the hierarchy of the battery structure that determines its functionality. An in-depth understanding of the battery function, degradation, and failure mechanisms requires a thorough and systematic investigation from structural, chemical, mechanical, and dynamic perspectives. Here we present a macro-to-nano zoom through the hierarchy of a commercial 18650 type LIB using a suite of state-of-the-art X-ray microscopy techniques. Damage, deformation, and heterogeneity at different length scales are visualized and are associated with different degradation phenomena and mechanisms. Our results highlight the importance of the mechanical properties of the cathode material, which could impact both the immediate and the long-term cell behaviors significantly. While this study focuses on a commercial lithium cell with a standard configuration, our findings can be extrapolated and are applicable to the development of next-generation energy storage technology, e.g. solid-state batteries.
\end{abstract}

\section{Introduction}

The development and commercialization of lithium-ion batteries has a tremendous impact on how energy is stored, transported, and utilized, which lies at the heart of the modern lifestyle, in which consumer electronics are ubiquitous and indispensable. ${ }^{1}$ With an exceptional energy density and lifespan, LIBs are one of the most important portable power sources, which not only have a very significant and growing market share but are also rapidly broadening their use cases, e.g. for powering electric vehicles and for operation under extreme conditions. ${ }^{2,3}$ These new application scenarios, in turn, led to a broad range of development efforts spanning from raw material research to cell design and system engineering. ${ }^{4}$

Although the "rocking-chair-type" chemical reaction in today's LIBs still follows the same principle since LIBs were first invented, over the past few decades, researchers in this field have developed a rather comprehensive understanding of the structure-chemistry-performance interplay., ${ }^{5,6}$ We point out here that this research field largely benefits from the rapid developments in materials characterization techniques, e.g. electron or synchrotron based diffraction, spectroscopy, and

\footnotetext{
${ }^{a}$ Stanford Synchrotron Radiation Lightsource, SLAC National Accelerator Laboratory, Menlo Park, CA 94025, USA. E-mail: liuyijin@slac.stanford.edu

${ }^{b}$ ESRF-The European Synchrotron, Grenoble 38043, France

'Sigray Inc., Concord, CA 94520, USA

$\dagger$ Electronic supplementary information (ESI) available. See DOI: $10.1039 / \mathrm{d} 1 \mathrm{ta0} 2262 \mathrm{~h}$

¥ G. Zan, J. Zhang, and F. Monaco contributed equally to this work.
}

microscopy tools, which probe different materials properties that are important to the battery performance. ${ }^{6-10}$ For example, electron microscopy is often utilized to image the battery electrode material with very high spatial resolution. Scanning electron microscopy (SEM) has been very successful in imaging the LIB electrode micro-morphology that is determined by the local spatial arrangements of the active and the inactive materials, e.g. $\mathrm{LiNi}_{x} \mathrm{Mn}_{y} \mathrm{Co}_{z} \mathrm{O}_{2}$ (NMC) cathode particles embedded in a porous carbon and binder matrix. ${ }^{11}$ Combined with a focused ion beam (FIB), SEM can further image the electrode or particle cross-sections, building up a three-dimensional image layer by layer. ${ }^{12}$ Scanning transmission electron microscopy (STEM) can further go down to spatial resolution at the atomic-level for visualizing the localized lattice configuration and cation mixing effect, which affects the lithium diffusion kinetics at the nanoscale. ${ }^{13}$ The X-ray diffraction technique, on the other hand, probes the bulk-averaged crystalline structure in the reciprocal space by fingerprinting diffraction peaks to different crystalline phases. This method has been utilized to capture the dynamic evolution of the battery cathode lattice structure, including reversible lattice breathing ${ }^{\mathbf{1 4}}$ and irreversible phase transformations $^{\mathbf{1 5 , 1 6}}$ that occur under operating conditions. Synchrotron spectroscopic tools shows sensitivity to the chemical states of the battery materials with exceptional specificity to targeted element(s) of interest. Synchrotron spectroscopy has been broadly applied to understand and gain information on the development of new doping/coating chemistry for battery material synthesis. ${ }^{\mathbf{1 7}}$ 
To systematically study the unique hierarchical structure of a practical LIB cell, a multi-scale, multi-contrast, highdimensional, and non-destructive imaging approach is highly desirable. ${ }^{6}$ This is because a real-world battery performance decay process is highly complicated, featuring both acute and chronic degradation mechanisms that are associated with structural and chemical complexities at different length scales. For example, LIB thermal runaway is a faulty process that involves a positive temperature feedback and leads to an unstoppable chain reaction. When thermal runaway is triggered, the temperature increases and the battery releases its energy very rapidly, causing a fire within a very short period of time. ${ }^{18}$ Although thermal runaway itself is a catastrophic phenomenon and it affects the entire cell dramatically, its fundamental causes are often localized and could have existed in the cell for a long time (even since the cell was manufactured). One of the most common reasons for LIB thermal runaway is internal short circuit, which could be associated with pre-existing cell defects or undesired local side reactions, e.g. lithium dendrite growth. ${ }^{\mathbf{1 9 2 0}}$ Early detections of these multiscale defective features could help prevent and mitigate degradation.

In this work, we present a macro-to-nano zoom through the hierarchy of a commercial LIB using a suite of state-of-the-art Xray microscopy techniques, including laboratory microtomography, synchrotron phase-contrast holotomography, and synchrotron nano-resolution spectro-tomography. We visualize and quantify damage, deformation, and heterogeneity in the studied LIB cell at different length scales and associate them with different degradation mechanisms. Non-destructive threedimensional (3D) imaging at the cell-level not only provides an overview of our 18650 type cylindrical cell, but also allows us to identify defective regions for high-resolution synchrotron imaging studies. We report that the electrode deformation and delamination are closely associated with active particle cracking. The sequential development of intragranular and intergranular chemical heterogeneities is observed and is correlated with the degree of particle disintegration. Our results highlight the importance of the chemomechanical properties of the active cathode material, which directly modulate the local chemistry at the nano-to-meso scale as well as the macroscopic behaviours at the electrode-to-cell level. The demonstrated multi-scale, multi-contrast, and high-dimensional X-ray imaging approach is broadly applicable to general material studies beyond the field of LIB research.

\section{Methods}

\subsection{Laboratory X-ray microscopy}

Laboratory X-ray microscopy (XRM) was utilized for visualizing the interior microstructures of Li-ion batteries. ${ }^{21-23}$ Initial investigations of the 18650 battery cell were conducted using a Sigray PrismaXRM ${ }^{\mathrm{TM}} \mathrm{X}$-ray microscope (Sigray inc., Concord, CA, USA), equipped with a flat panel detector, which comprised $2940 \times 2304$ pixels with a pixel size of $49.5 \mu \mathrm{m}$. The entire width of the 18650 type cylindrical battery cell was captured in a single field of view (FOV) with a magnification of 4.9 in a scan of $\sim 2.5 \mathrm{~h}$. Four tomographic scans were carried out with the cell translated along the vertical direction to cover the entire height of the 18650 battery. The X-ray source was operated at a voltage of $120 \mathrm{kV}$ and a current of $0.1 \mathrm{~mA}$. In every tomography, 4801 projection images were recorded over an angular range of $360^{\circ}$. These projection images were subsequently reconstructed using a Feldkamp-Davis-Kress (FDK) algorithm, which produces a 3D isotropic voxel size of $10 \mu \mathrm{m}$ over the entire FOV covering the 18650 battery cell in its entirety. The resulting four tomographic volumes were then stitched together and visualized using an Avizo (Thermo Fisher Scientific, Waltham, Massachusetts, USA). In addition to performing tomography with a large FOV, the PrismaXRM ${ }^{\mathrm{TM}}$ system also offers the capability to nondestructively probe a smaller region of interest with higherresolution analysis based on a unique optics-based design, an approach also known as local tomography. ${ }^{24,25}$ With a region of interest identified in the overview scan with a coarse resolution, we zoom into image with a finer voxel size of $1.2 \mu \mathrm{m}$. For further synchrotron imaging with nanoscale resolution, the 18650 battery cell was dismantled and unrolled in an argon-filled glovebox. We isolated specimens from the identified regions of interest for synchrotron experiments, which will be described below.

\subsection{Synchrotron phase-contrast holotomography}

Synchrotron X-ray phase contrast imaging (XPCI) generates image contrast stemming from the X-ray phase shift induced by the sample, which could provide substantially improved sensitivity for the measurements, in particular for the light (low-Z) materials. ${ }^{26}$ Hence both heavy metal oxides (the NMC particles) and the porous carbon and binder matrix can be identified by using the hard XPCI with adequate quantitative sensitivity. ${ }^{27,28}$ To investigate the morphological study of the $\mathrm{Li}$ battery cathode with high spatial resolution down to the nanoscale, X-ray phase contrast holotomography measurements were performed at the ID16A-NI nano-imaging beamline at the European Synchrotron Radiation Facility (ESRF) in Grenoble, France. This beamline features a high-energy hard X-ray nano-probe, which delivers a brilliant flux (up to $10^{12}$ photons per s at $\Delta E / E \approx 1 \%$ ) within a nano-focus down to $\sim 20 \mathrm{~nm} \cdot{ }^{29}$ Its unique performance was implemented with two pairs of advanced multilayer-coated Kirkpatrick-Baez (KB) optics, operating at 17 and $33.6 \mathrm{keV}$ respectively. Our battery cathode sample was placed downstream of the $\mathrm{KB}$ focus under the illumination of $33.6 \mathrm{keV}$ X-rays. Magnified radiographs were acquired using an X-ray detector equipped with a FReLoN charged-coupled device with a $2048 \times 2048$ binned pixels array. The large geometry magnification of the cone beam contributes to the effective pixel size at $\sim 70 \mathrm{~nm}$. One entire phase contrast nano-tomographic volume was retrieved from four tomograms, which were obtained at four different focus-to-sample distances. For every single tomography acquisition, 2000 projections over $180^{\circ}$ were recorded with $0.15 \mathrm{~s}$ exposure time. These collected tomographic projection data sets were then used as an input for the phase retrieval to create two dimensional (2D) phase maps in all angular projections. ${ }^{30}$ The 
retrieved $2 \mathrm{D}$ phase maps subsequently underwent a tomographic reconstruction procedure using a filtered back projection algorithm (ESRF PyHST software package). ${ }^{31}$ The resulting $3 \mathrm{D}$ phase contrast volumes are proportional to the real part of the complex refractive index, which is governed by the local distribution of the electron density in the sample.

\subsection{Nano-resolution X-ray spectro-microscopy}

In addition to improvements in X-ray brightness, synchrotrons are also equipped to offer X-ray beam energy tunability by using crystal monochromators. This capability facilitates a hybrid of imaging and spectroscopy, which is termed X-ray spectromicroscopy. ${ }^{32}$ This approach can be used to evaluate the morphology of the materials with compositional and chemical characteristics. We carried out the 3D spectro-tomography experiments using transmission X-ray microscopy (TXM) at the beamline 6-2c of the Stanford Synchrotron Radiation Lightsource (SSRL) of SLAC National Accelerator Laboratory. The particles were carefully peeled off from the cathode of the 18650 type battery and loaded in a quartz capillary. During 3D Xray absorption near edge structure (XANES) measurements, tomography was carried out at 63 different energy points spanning across the nickel (Ni) K-edge. The entire energy region ranges from $8178 \mathrm{eV}$ to $8570 \mathrm{eV}$. The energy step is set to $1 \mathrm{eV}$ in the near edge region to ensure the energy resolution. In preedge and post-edge regions, the energy step was set to $10 \mathrm{eV}$ and $20 \mathrm{eV}$, respectively, for covering a relatively wide energy window for spectra normalization. For every tomography, the specimen was rotated from $-90^{\circ}$ to $90^{\circ}$ with an angular step size of $1^{\circ}$. The $3 \mathrm{D}$ tomographic reconstruction and spectroscopic data analysis were carried out using an in-house developed software package known as TXM-Wizard. ${ }^{33}$

\section{Results and discussion}

In collaboration with our industry partners, here we carry out a systematic investigation on an 18650 type cylindrical LIB cell that failed the factory line's quality control inspection (QCI) due to a self-discharging effect (see the ESI $\dagger$ for more details). Industrial QCI often looks into the electrochemical signatures of the cell, which renders the cell behaviours at a macroscopic scale. Industrial tomography has been used for LIB cell inspections, but there is generally a trade-off between spatial resolution and the throughput of the tomographic imaging experiments. Therefore, an in-depth and high-resolution study of defective cells is highly desirable but is not broadly adopted in industrial production settings.

\subsection{Cell inspection using $X$-ray microtomography}

In our study, we first obtain an X-ray overview of the whole cell using the microtomography technique (more details can be found in the Methods section). As shown in Fig. 1, the jelly roll

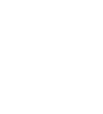

a

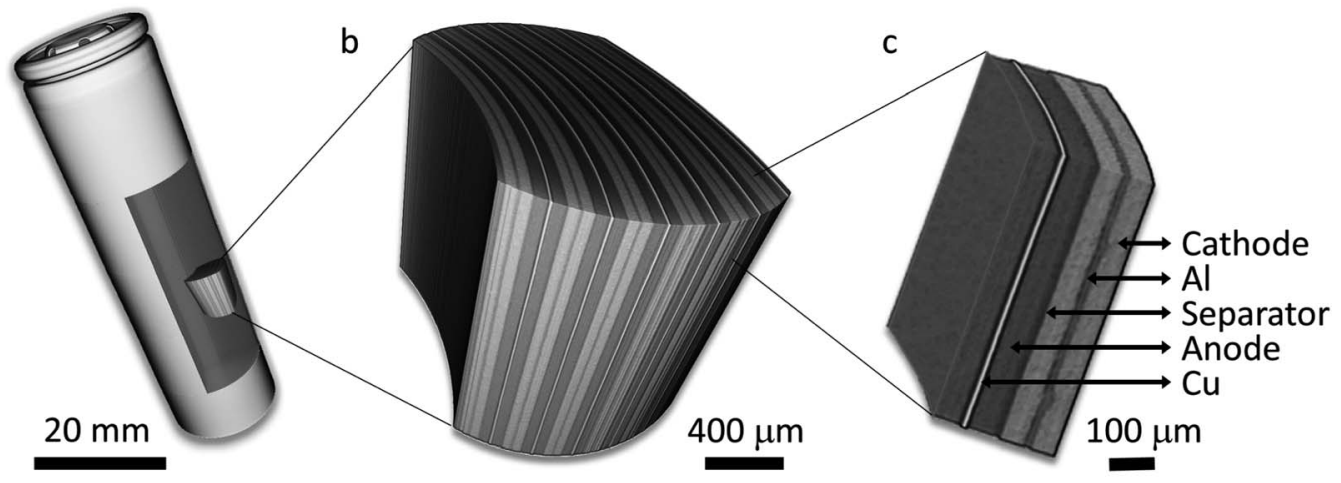

d

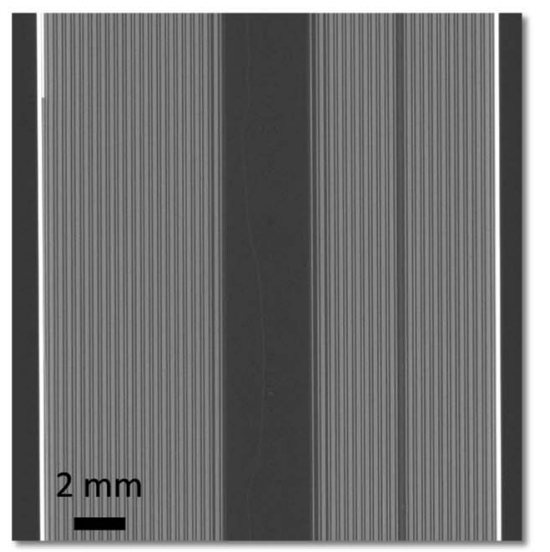

e

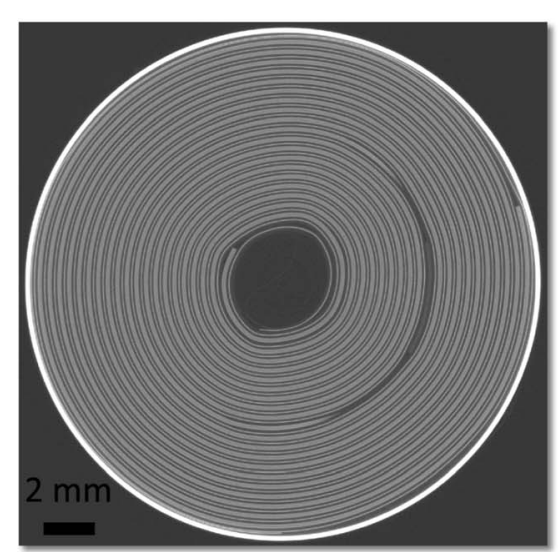

Fig. 1 The cell-level structural complexity in a commercial 18650 type LIB. Panels (a) to (c) are the 3D visualization of the microtomographic data of the cell acquired with different settings at different resolutions. Panels (d) and (e) are $2 \mathrm{~d}$ vertical slices of the reconstructed volume. 
a

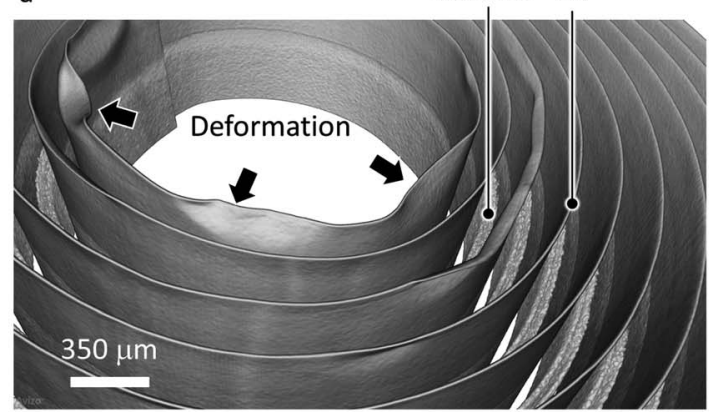

c

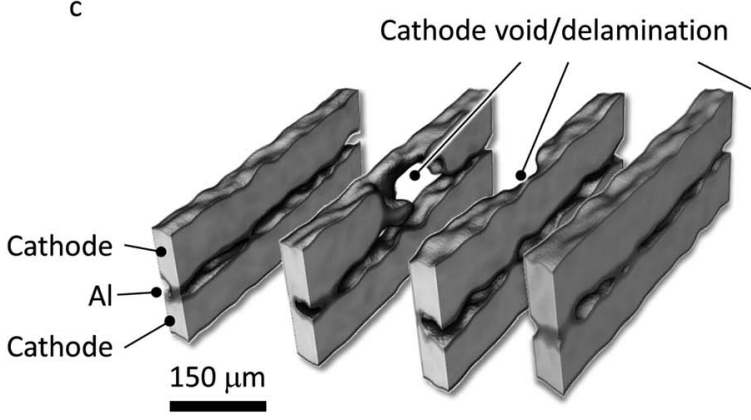

b

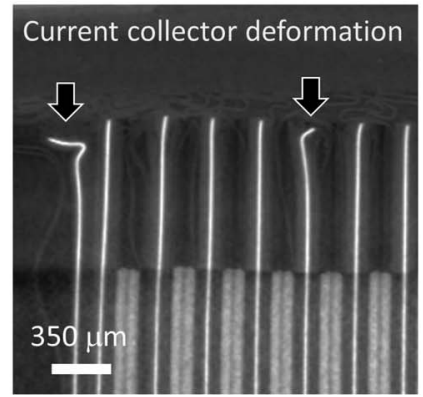

d

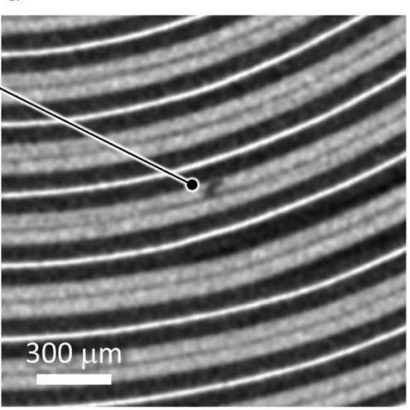

Fig. 2 The identified defects in a commercial 18650 type LIB. Panels (a) and (b) are 3D and 2D visualizations of the deflected Cu current collector, respectively. Panels (c) and (d) are the 3D and 2D visualizations of a defective region on the NMC cathode, with the void/delamination clearly observed.

structure of the cell can be clearly visualized. In the tomographic data, the cathode, which consists of $\mathrm{LiNi}_{0.5} \mathrm{Mn}_{0.3} \mathrm{Co}_{0.2^{-}}$ $\mathrm{O}_{2}$ (NMC532), and the anode $\mathrm{Cu}$ current collector show strong contrast, while the graphite anode and the cathode Al current collector are relatively transparent (see Fig. 1c for details). Fig. 1d and e show 2D virtual slices in the vertical and horizontal orientations, respectively. The overview of the cell confirms that this is a typical 18650 type cylindrical cell with a standardized cell configuration.

We further carried out a careful inspection of the microtomography data and identified several defective regions in the cathode. For example, a zoom-in view of the electrode near the positive terminal (Fig. 2a) shows a significant deflection of the $\mathrm{Cu}$ current collector, which could pierce though the separator and cause a short circuit if a physical contact between the current collectors for cathode and anode is established. The deformation of the $\mathrm{Cu}$ current collector is more obvious in the virtual slice shown in Fig. $2 \mathrm{~b}$, featuring a tightened and irregularly shaped gap between the $\mathrm{Cu}$ and $\mathrm{Al}$ current collectors. In the electrode body, overall, the composite cathode electrode in this cell is rather uniform with an averaged thickness at around $60 \mu \mathrm{m}$, which is quite standard for industrial 18650 type cells. Here we point out that academic battery research often utilizes thin electrodes, which is fine for fundamental research; however, there is a strong practical incentive to increasing the electrode thickness for an increased energy density at the cell level. Nonetheless, there are a number of drawbacks associated with thick electrodes, e.g. a more severe cell polarization effect and potentially reduced mechanical robustness. ${ }^{34}$ In our data, we observed a clear electrode delamination effect at a few different locations and a selected example is visualized in 3D and $2 \mathrm{D}$ in Fig. 2c and d, respectively. The electrode delamination could have a profound impact, involving both immediate and long-term degradations. Specifically, the formation of void/ delamination in the cathode could lead to a reduced capacity, accelerate undesired side reactions at the fresh solid-liquid interface, and an increase in local electrical and ionic resistance, which could be exothermic.

\subsection{Association of cathode delamination with particle disintegration}

To gain more insights into the cathode deformation and delamination effect, we disassembled the cylindrical cell in an argon-filled glovebox and harvested the region of interest as identified by our tomographic overview of the cell. The retrieved electrode was then scanned using synchrotron-based nanoresolution holotomography. More details can be found in the Methods section. In our experiment, the holotomography technique offers a spatial resolution at 70-100 nm with quantitative phase retrieval, which allows us to visualize and quantify the delaminated regions in greater detail. As shown in Fig. 3a and $b$, the cathode of this cell is made of irregularly shaped secondary particles at 3-4 $\mu \mathrm{m}$. This is relatively small compared to the commonly used spherical secondary particles at $\sim 10 \mu \mathrm{m}$. Part of the reason is that this cell is intended for fast-charging applications, which prefer active particles with a small size for reducing the lithium diffusion length and promoting the reaction kinetics during the fast-charging process. These NMC particles are fairly closely packed, and their degree of fracturing 


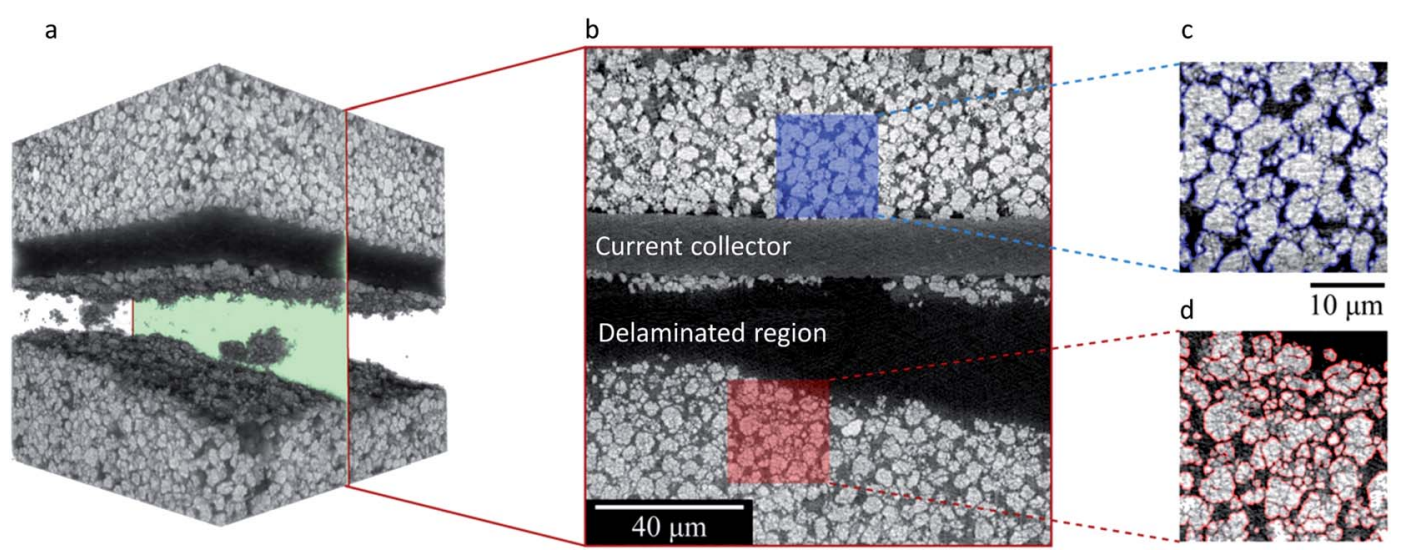

Fig. 3 Synchrotron-based holotomography on a delaminated cathode electrode. Panel (a) shows the 3D visualization of the damaged region. Panel (b) shows a selected virtual slice through the center of the volume with two regions of interest enlarged for visualization in panel (c) and (d). Although the blue region is still intact, some mild particle disintegration can already be observed. The red region, on the other hand, is severely damaged.

is quite heterogeneous (Fig. $3 \mathrm{c}$ and d). It is interesting to report that the delaminated region is associated with more severe particle cracking. This can be directly visualized in the magnified view in Fig. 3d. We further conduct a quantification of the particle size distribution over the delaminated region (Fig. 4). Fig. 4 displays the raw image of the particle fragment segmentation and depth-dependent region segmentation (regions 1 to 5 ) in the left panel. The particle fragment size distribution over these regions at different depths is quantified and shown in the right panel. It is clear from our data that the delamination is not caused by a lack of cohesion between the NMC electrode and the $\mathrm{Al}$ current collector. Instead, the fracturing of the active particles appears to be the root cause of the mechanical weakness, which then results in the observed electrode delamination.
It has been broadly reported that, while it is ultimately induced by the cathode lattice breathing upon electrochemical cycling, the cathode particle fracturing is highly heterogeneous and can be affected by a number of different factors, including the local morphology, the electrochemical conditions, and the temperature. The observation of the asymmetry in Fig. 3, showing the delamination on only one side of the electrode, echoes this point very well. Compared to the delamination phenomenon, the particle cracking is more ubiquitous throughout the entire cell. Therefore, the electrode delamination is triggered by the intensification of the agglomerated particle cracking effect, which could be caused by large local current density that is detrimental to the cell stability and safety.

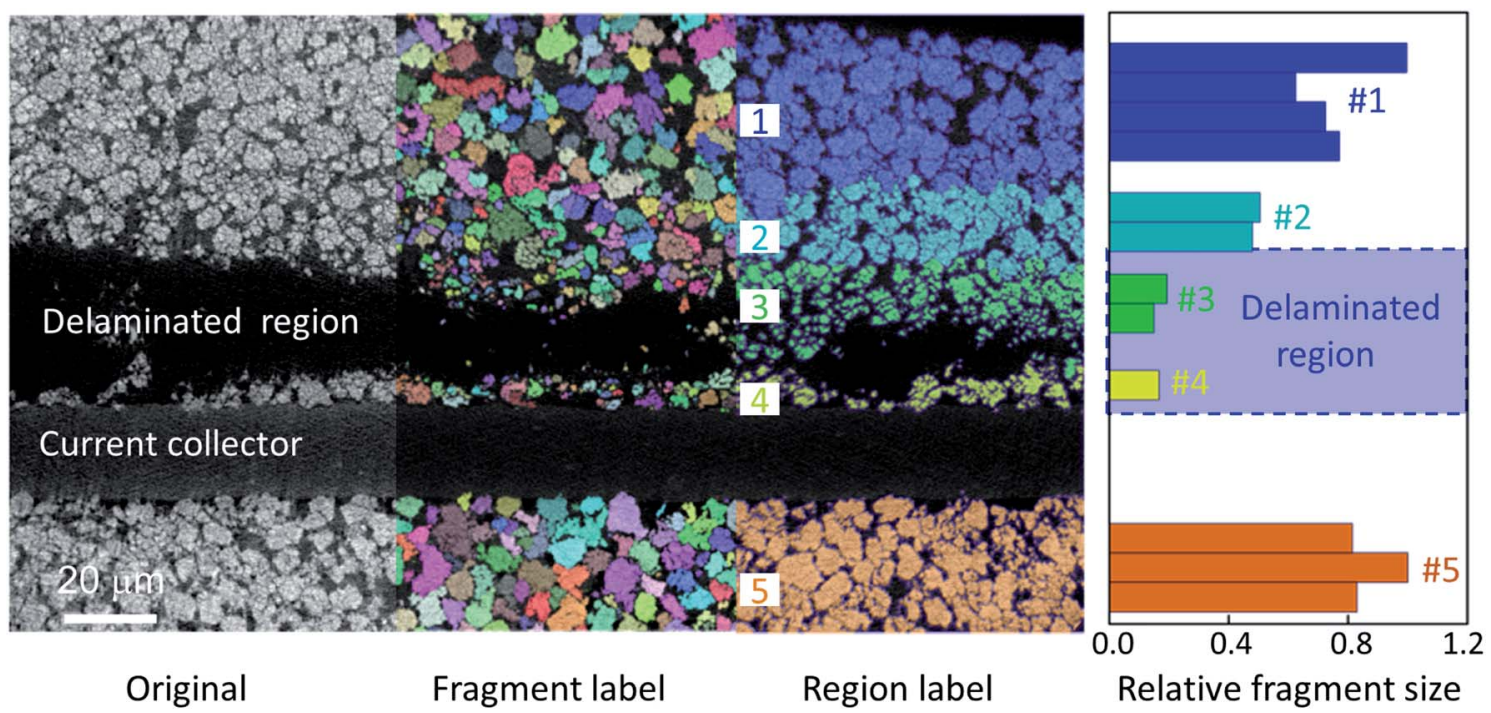

Fig. 4 Quantification of the particle size distribution over a select region. A clear association between the delamination effect and the cracking of active NMC particles is observed. 


\subsection{Nanoscale inter-granular and inter-granular chemical heterogeneity}

It is a consensus that the mechanical damage of the battery cathode has profound chemical consequences. While our highresolution holotomographic visualization of the delaminated cathode electrode features a vastly different degree of particle fracturing at different locations, it requires a nano-resolution probe with chemical sensitivity to further unveil the localized chemomechanical interplay. For this purpose, we turn to nanoresolution spectro-microscopy for an in-depth analysis of the inter-granular and intra-granular chemical heterogeneity.

In our spectro-microscopy experiment, we combined the nano-resolution tomographic scan with an energy scan across the Ni K-edge for fingerprinting the local oxidation state of Ni. $\mathrm{Ni}$ is the most redox active cation in the NMC cathode. The valence state of $\mathrm{Ni}$ has been broadly utilized as a proxy for the local state of charge (SOC) ${ }^{35}$ With nano-resolution spectrotomography over the Ni edge, the SOC heterogeneity (Fig. 5d) can be resolved and correlated with the local morphology (Fig. 5a). As shown in Fig. 5a, the imaged volume contains several particles and broken particle fragments. We first carried out a conventional segmentation process for labelling all the isolated fragments (Fig. 5b). Because the cathode particles exhibit very different degrees of cracking, the identified fragments are quite different in their respective volumes. We further plot the fragment mean SOC (mean value of the Ni Kedge energy) versus their respective volumes in Fig. $5 \mathrm{c}$ and separate these fragments into three groups based on their respective size. Statistical analysis of this data suggests that the small fragments (region I in Fig. 5c) are rather scattered in their SOC distribution, with the averaged value being slightly higher than that of the medium-sized (region II) and large (region III) fragments. Larger fragments are generally more stable in their SOC, which is in good agreement with the conventional wisdom because it will take a lot of charge transfer and lithium diffusion to alter the averaged SOC of a large piece of a NMC particle (fragment).

The exceptional image quality allows us to not only analyse the detached particle fragments, but also to identify every single primary grain in the imaged volume (see illustration in Fig. 5e). With this segmentation result, we further plot the internal SOC heterogeneity of the grains, which is defined by the standard deviation of the Ni K-edge energy over the grain (see illustration in Fig. S3 in ESI $\dagger$ ), versus the corresponding averaged grain SOC (see Fig. $5 \mathrm{f}$ ). The absolute value in the vertical axis of Fig. $5 \mathrm{f}$ is associated with the intra-granular SOC heterogeneity and the spread of the data points in the horizontal direction features the inter-granular SOC heterogeneity. We categorized all the grains based on the degree of the damage in the corresponding a

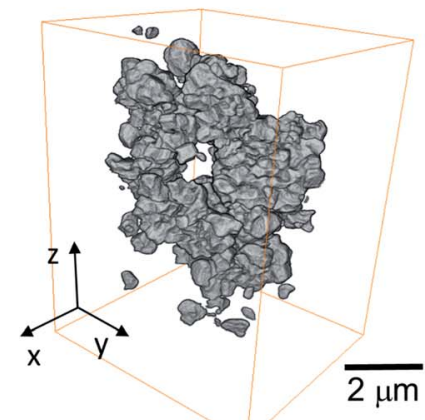

b

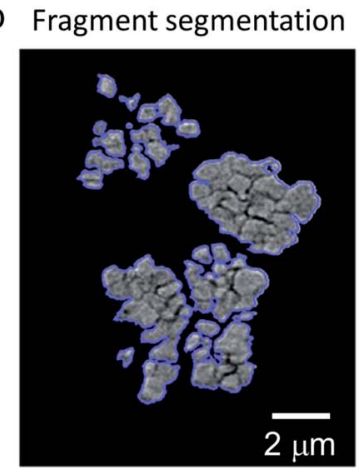

d 3D XANES

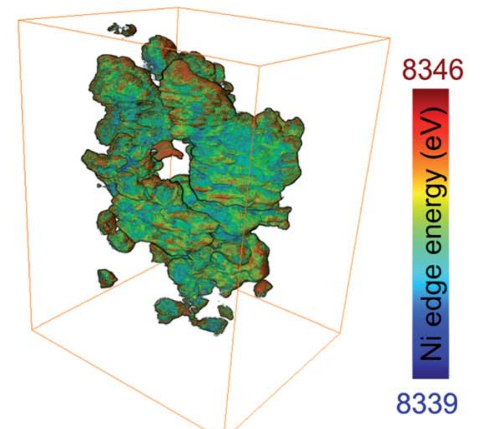

e

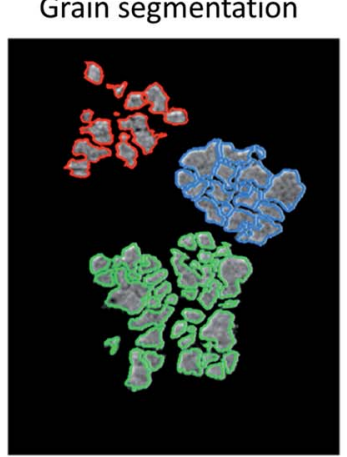

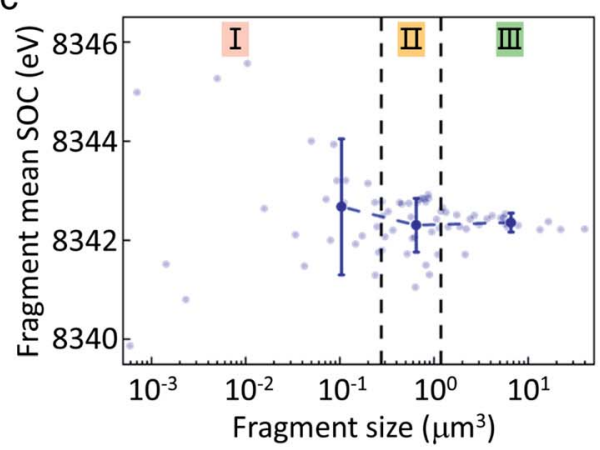

f

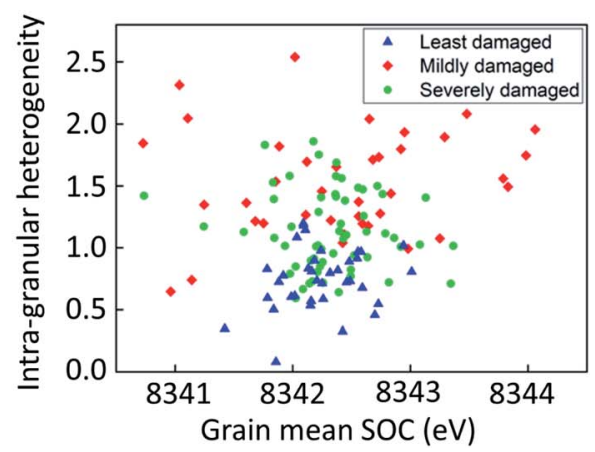

Fig. 5 Nano-resolution spectro-tomography on a cluster of cathode particles with different degrees of cracking. Panel (a) shows the 3D morphology of the particle-cluster. Panel (b) shows the segmentation result of particle fragments in a 2D slice. Panel (c) shows the plot of the mean SOC of the fragments (using the Ni edge energy as a proxy) versus their respective volumes. Panel (d) shows the 3D XANES result, highlighting the heterogeneity in the Ni edge energy. Panel (e) shows the segmentation of every single grain in the imaged volume. Panel (f) shows the plot of the inter-granular heterogeneity of the grains, which is measured by the standard deviation of the Ni edge energy, versus their respective mean SOC. 
secondary particles and color-coded the data points to blue (for grains from least damaged secondary particles), green (for grains from mildly damaged secondary particles), and red (for grains from severely damaged secondary particles). The grains from rather intact secondary particles (blue data points in Fig. 5f) show a relatively low level of inter-granular and intragranular SOC heterogeneities. Accompanied by a mild secondary particle damage, there is a noticeable upward shift of the green data points while the horizontal spread is not significantly impacted (compared with the blue data points). This suggests that the intra-granular SOC heterogeneity first develops upon secondary particle fracturing and is followed by the inter-granular SOC heterogeneity, which is amplified in the later stage of the particle cracking. While the inter-granular SOC heterogeneity maybe more directly associated with the polarization effect and the capacity fade at the cell-level, the intragranular SOC heterogeneity appears to be a more fundamental process that develops at the mildly damage stage of the cathode degradation. Recently, single-crystal NMC particles are being actively studied as they could offer improved mechanical robustness with suppressed cracking damages. ${ }^{36,37}$ This approach is well aligned with our observation. Focusing on the intra-granular reaction kinetics, we point out that trace doping with elements of low solubility could lead to dopant segregation and, subsequently, impact the lithium diffusion pathways. ${ }^{\mathbf{1 3 , 3 8 - 4 0}}$ This method can be utilized to effectively tailor the mesoscale SOC heterogeneity for a balanced surface stability and diffusion efficiency.

\section{Conclusions}

LIBs have a very complicated hierarchical structure. Realization of their functionality as an energy reservoir requires multiple structural components to be involve in rather complicated chemical reactions that are highly heterogeneous across a wide range of length scales. Although the high-level conceptual design of a battery cell has remained the same for many decades, we are witnessing very significant improvements in battery performance that are achieved by tremendous engineering efforts that tackle the structure and chemical complexities in batteries. In this work, we combined a suite of cutting-edge imaging techniques to reveal the structural defects in a commercial LIB cell. We associate the electrode delamination/deformation to the fracturing of the active cathode material. Finally, we looked into the microscopic chemomechanical interplay by quantifying the inter-granular and intra-granular SOC heterogeneities in over a hundred primary grains that are associated with different degrees of secondary particle disintegration. We concluded that the intra-granular SOC heterogeneity is developed in the mildly damage stage and could be a fundamental process that accelerates the particle disintegration. Our work also echoes recent efforts in utilizing single-crystal particles as building blocks for composite cathode electrodes. This work features a multi-scale, multi-modality, and high-dimensional X-ray imaging approach, which is broadly applicable to fundamental and applied research activities.

\section{Author contributions}

Y. Liu conceived the research. G. Zan, J. Zhang, F. Monaco, S. Gul, G. Qian, and J. Li performed the experiments. G. Zan, J. Zhang, F. Monaco, and Y. Liu analyzed the data. D. J. Vine, P. Cloetens, W. Yun, and P. Pianetta contributed to the interpretation of the experimental data. G. Zan and Y. Liu prepared the manuscript with critical inputs from all the authors.

\section{Conflicts of interest}

There are no conflicts to declare.

\section{Acknowledgements}

The use of the Stanford Synchrotron Radiation Lightsource, SLAC National Accelerator Laboratory, was supported by the U.S. Department of Energy, Office of Science, Office of Basic Energy Sciences under Contract No. DE-AC02-76SF00515. Part of this work was performed at the Stanford Nano Shared Facilities (SNSF), supported by the National Science Foundation under the award ECCS-1542152. The engineering support from D. Van Campen, D. Day, and V. Borzenets for the TXM experiment at beamline 6-2C of SSRL is gratefully acknowledged. The phase contrast holotomography experiment was performed at the beamline ID16A of the European Synchrotron Radiation Facility (ESRF), Grenoble, France, in the frame of proposal MA4626.

\section{References}

1 M. Li, J. Lu, Z. Chen and K. Amine, Adv. Mater., 2018, 30, 1800561.

2 Z. P. Cano, D. Banham, S. Ye, A. Hintennach, J. Lu, M. Fowler and Z. Chen, Nat. Energy, 2018, 3, 279-289.

3 N. Nitta, F. Wu, J. T. Lee and G. Yushin, Mater. Today, 2015, 18, 252-264.

4 Y. Ding, Z. P. Cano, A. Yu, J. Lu and Z. Chen, Electrochem. Energy Rev., 2019, 2, 1-28.

5 S. Xia, L. Mu, Z. Xu, J. Wang, C. Wei, L. Liu, P. Pianetta, K. Zhao, X. Yu, F. Lin and Y. Liu, Nano Energy, 2018, 53, 753-762.

6 F. Lin, Y. Liu, X. Yu, L. Cheng, A. Singer, O. G. Shpyrko, H. L. Xin, N. Tamura, C. Tian, T.-C. Weng, X.-Q. Yang, Y. S. Meng, D. Dordlund, Y. Wanli and M. M. Doeff, Chem. Rev., 2017, 117, 13123-13186.

7 Y. Yuan, K. Amine, J. Lu and R. Shahbazian-Yassar, Nat. Commun., 2017, 8, 15806.

8 C. Cao, M. F. Toney, T.-K. Sham, R. Harder, P. R. Shearing, X. Xiao and J. Wang, Mater. Today, 2019, 34, 132-147.

9 S.-M. Bak, Z. Shadike, R. Lin, X. Yu and X.-Q. Yang, NPG Asia Mater., 2018, 10, 563-580.

10 D. Liu, Z. Shadike, R. Lin, K. Qian, H. Li, K. Li, S. Wang, Q. Yu, M. Liu and S. Ganapathy, Adv. Mater., 2019, 31, 1806620.

11 R. Xu, L. S. De Vasconcelos, J. Shi, J. Li and K. Zhao, Exp. Mech., 2018, 58, 549-559. 
12 M. M. Besli, S. Xia, S. Kuppan, Y. Huang, M. Metzger, A. K. Shukla, G. Schneider, S. Hellstrom, J. Christensen, M. M. Doeff and Y. Liu, Chem. Mater., 2019, 31, 491-501.

13 G. Qian, H. Huang, F. Hou, W. Wang, Y. Wang, J. Lin, S.-J. Lee, H. Yan, Y. S. Chu and P. Pianetta, Nano Energy, 2021, 105926.

14 C. Tian, D. Nordlund, H. L. Xin, Y. Xu, Y. Liu, D. Sokaras, F. Lin and M. M. Doeff, J. Electrochem. Soc., 2018, 165, A696.

15 E. Hu, X. Wang, X. Yu and X.-Q. Yang, Acc. Chem. Res., 2018, 51, 290-298.

16 K. W. Nam, S. M. Bak, E. Hu, X. Yu, Y. Zhou, X. Wang, L. Wu, Y. Zhu, K. Y. Chung and X. Q. Yang, Adv. Funct. Mater., 2013, 23, 1047-1063.

17 A. M. Wise, C. Ban, J. N. Weker, S. Misra, A. S. Cavanagh, Z. Wu, Z. Li, M. S. Whittingham, K. Xu, S. M. George and M. F. Toney, Chem. Mater., 2015, 27, 6146-6154.

18 D. P. Finegan, M. Scheel, J. B. Robinson, B. Tjaden, I. Hunt, T. J. Mason, J. Millichamp, M. Di Michiel, G. J. Offer, G. Hinds, D. J. L. Brett and P. R. Shearing, Nat. Commun., 2015, 6, 6924.

19 K. J. Harry, D. T. Hallinan, D. Y. Parkinson, A. A. MacDowell and N. P. Balsara, Nat. Mater., 2014, 13, 69-73.

20 D. Wang, W. Zhang, W. Zheng, X. Cui, T. Rojo and Q. Zhang, Adv. Sci., 2017, 4, 1600168.

21 J. Gelb, D. P. Finegan, D. J. L. Brett and P. R. Shearing, J. Power Sources, 2017, 357, 77-86.

22 P. R. Shearing, N. P. Brandon, J. Gelb, R. Bradley, P. J. Withers, A. J. Marquis, S. Cooper and S. J. Harris, J. Electrochem. Soc., 2012, 159, A1023.

23 M. D. R. Kok, J. B. Robinson, J. S. Weaving, A. Jnawali, M. Pham, F. Iacoviello, D. J. L. Brett and P. R. Shearing, Sustainable Energy Fuels, 2019, 3, 2972-2976.

24 M. Feser, J. Gelb, H. Chang, H. Cui, F. Duewer, S. H. Lau, A. Tkachuk and W. Yun, Meas. Sci. Technol., 2008, 19, 094001.

25 A. P. Merkle and J. Gelb, Microsc. Today, 2013, 21, 10-15.

26 Y. Liu, J. Nelson, C. Holzner, J. C. Andrews and P. Pianetta, J. Phys. D: Appl. Phys., 2013, 46, 494001.

27 Y. Yang, R. Xu, K. Zhang, S. J. Lee, L. Mu, P. Liu, C. K. Waters, S. Spence, Z. Xu, C. Wei, D. J. Kautz, Q. Yuan, Y. Dong, Y.-S. Yu, X. Xiao, H.-K. Lee, P. Pianetta, P. Cloetens,
J.-S. Lee, K. Zhao, F. Lin and Y. Liu, Adv. Energy Mater., 2019, 9, 1900674.

28 Z. Jiang, J. Li, Y. Yang, L. Mu, C. Wei, X. Yu, P. Pianetta, K. Zhao, P. Cloetens, F. Lin and Y. Liu, Nat. Commun., 2020, 11, 2310.

29 J. C. Da Silva, A. Pacureanu, Y. Yang, S. Bohic, C. Morawe, R. Barrett and P. Cloetens, Optica, 2017, 4, 492-495.

30 P. Cloetens, W. Ludwig, J. Baruchel, D. Van Dyck, J. Van Landuyt, J. Guigay and M. Schlenker, Appl. Phys. Lett., 1999, 75, 2912-2914.

31 A. Mirone, E. Brun, E. Gouillart, P. Tafforeau and J. Kieffer, Nucl. Instrum. Methods Phys. Res., Sect. B, 2014, 324, 41-48.

32 F. Meirer, J. Cabana, Y. Liu, A. Mehta, J. C. Andrews and P. Pianetta, J. Synchrotron Radiat., 2011, 18, 773-781.

33 Y. Liu, F. Meirer, J. Wang, G. Requena, P. Williams, J. Nelson, A. Mehta, J. C. Andrews and P. Pianetta, Anal. Bioanal. Chem. Res., 2012, 404, 1297-1301.

34 J. Liu, Z. Bao, Y. Cui, E. J. Dufek, J. B. Goodenough, P. Khalifah, Q. Li, B. Y. Liaw, P. Liu, A. Manthiram, Y. S. Meng, V. R. Subramanian, M. F. Toney, V. V. Viswanathan, M. S. Whittingham, J. Xiao, W. Xu, J. Yang, X.-Q. Yang and J.-G. Zhang, Nat. Energy, 2019, 4, 180-186.

35 Z. Xu, Z. Jiang, C. Kuai, R. Xu, C. Qin, Y. Zhang, M. M. Rahman, C. Wei, D. Nordlund, C.-J. Sun, X. Xiao, X.-W. Du, K. Zhao, P. Yan, Y. Liu and F. Lin, Nat. Commun., 2020, 11, 83.

36 G. Qian, Y. Zhang, L. Li, R. Zhang, J. Xu, Z. Cheng, S. Xie, H. Wang, Q. Rao, Y. He, Y. Shen, L. Chen, M. Tang and Z.-F. Ma, Energy Storage Mater., 2020, 27, 140-149.

37 Y. Bi, J. Tao, Y. Wu, L. Li, Y. Xu, E. Hu, B. Wu, J. Hu, C. Wang and J.-G. Zhang, Science, 2020, 370, 1313-1317.

38 J. Zhang, Q. Li, C. Ouyang, X. Yu, M. Ge, X. Huang, E. Hu, C. Ma, S. Li, R. Xiao, W. Yang, Y. Chu, Y. Liu, H. Yu, X. Yang, X. Huang, L. Chen and H. Li, Nat. Energy, 2019, 4, 594-603.

39 Y. Hong, X. Huang, C. Wei, J. Wang, J.-N. Zhang, H. Yan, Y. S. Chu, P. Pianetta, R. Xiao and X. Yu, Chem, 2020, 6, 2759-2769.

40 W. H. Kan, B. Deng, Y. Xu, A. K. Shukla, T. Bo, S. Zhang, J. Liu, P. Pianetta, B.-T. Wang, Y. Liu and G. Chen, Chem, 2018, 4, 2108-2123. 